\title{
Lack of Cell Surface Fas/APO-1 Expression in Pulmonary Adenocarcinomas
}

Yoshihiro Nambu, ${ }^{\star}$ Steven J. Hughes, ${ }^{\ddagger}$ Alnawaz Rehemtulla, ${ }^{\S}$ Daniel Hamstra, ${ }^{\S}$ Mark B. Orringer, ${ }^{\ddagger}$ and David G. Beer ${ }^{\ddagger}$

$*$ Department of Internal Medicine, Division of Respiratory Diseases, Kanazawa Medical University, Ishikawa, Japan; and ${ }^{\ddagger}$ Department of Surgery, Section of Thoracic Surgery, and ${ }^{\S}$ Department of Radiation Oncology, University of Michigan Medical School, Ann Arbor, Michigan 48109

\section{Abstract}

The Fas receptor and ligand initiate an apoptotic pathway. Alterations in this pathway within tumor cells can result in escape from apoptosis and immune surveillance. We evaluated Fas protein expression in $\mathbf{4 2}$ primary pulmonary adenocarcinomas, and Fas expression and function in the lung adenocarcinoma cell lines A549 and A427. Immunohistochemical analysis demonstrated Fas protein expression in 47.6\% of the tumors; however, Fas-positive tumors demonstrated cytoplasmic staining without cell surface expression. Northern blot analysis indicated that levels of Fas mRNA were similar in Fas protein-positive tumors to levels in normal lung tissue, but were reduced in Fas protein-negative tumors. Soluble form Fas was not detected in the majority of these tumors either by RT-PCR or Western blot analysis. Cell surface Fas protein expression was minimal in A549 and A427 cell lines as determined by flow cytometry. Both cell lines demonstrated Fas mRNA expression by Northern blot analysis and abundant protein expression by Western blot analysis. Transfection of the Fas cDNA derived from A549 cells induced surface Fas protein in COS cells; however, stable transfection of a native Fas cDNA into A549 cells failed to induce surface Fas protein expression. Parental A549 cells and A549 cells transfected with a Fas expression vector were resistant to Fas-mediated apoptosis. Transgenic expression of a FLAG-tagged Fas cDNA in A549 cells, with visualization of the Fas-FLAG protein using confocal microscopy, demonstrated that the Fas-FLAG protein was retained within cytoplasmic portions of the cell and was not translocated to the cell surface. These findings suggest that the Fas protein is reduced or not present on the cell surface in the primary lung tumors and is sequestered within A549 tumorigenic lung cells, and these alterations directly affect the cells resistance to Fas-mediated apoptosis. (J. Clin. Invest. 1998. 101:1102-1110.) Key words: Fas/APO-1 • pulmonary adenocarcinoma • A549 • Fas-FLAG • posttranslational modification

Address correspondence to Dr. David G. Beer, B560 MSRB II, Box 0686, Department of Surgery, Section of Thoracic Surgery, University of Michigan Medical School, Ann Arbor, MI 48109. Phone: 313763-0325; FAX: 313-763-0323; E-mail: dgbeer@umich.edu

Received for publication 10 September 1997 and accepted in revised form 6 January 1998.

J. Clin. Invest.

(C) The American Society for Clinical Investigation, Inc. 0021-9738/98/03/1102/09 \$2.00

Volume 101, Number 5, March 1998, 1102-1110

http://www.jci.org

\section{Introduction}

Bronchogenic carcinoma represents the leading cause of malignancy-related mortality in the United States, and the prognosis of pulmonary adenocarcinoma is especially poor in advanced staged tumors despite improved efforts in earlier diagnosis and combination chemo- and radiation therapy (1). Apoptosis is a genetically encoded programmed cell death defined by distinct characteristic morphological and biochemical changes (2). In malignant cells, these physiological apoptotic pathways are often altered, resulting in a significant survival advantage. Tumor cells with developed resistance to apoptosis can survive despite immune system tumor surveillance and are often nonresponsive to antitumor treatments (3).

The Fas receptor (APO-1 or CD95) and ligand play a key role in the initiation of one apoptotic pathway (4). The loss of Fas protein has been reported to induce resistance to apoptosis; however, apoptotic resistance in some Fas-expressing malignant cells has also been reported (5). Three potential mechanisms for apoptotic resistance include: $(a)$ lack of cell-surface Fas protein expression; (b) overexpression of bcl-2 family proteins; and $(c)$ alterations in Fas intracellular signaling pathways (5). Fas protein can occur as both a cell-surface and a soluble protein, with the soluble form of Fas ( $\mathrm{sFas})^{1}$ protein being generated by alternative mRNA splicing (6-8). Four isoforms of sFas protein have been reported, with the predominant sFas protein resulting from deletion of the transmembranous domain coded by exon $6(6,8)$. This sFas can protect against Fasmediated apoptosis by neutralizing the Fas ligand (6). Elevated sFas protein levels in sera from patients with nonhematopoietic human malignancies has been reported (9), with the elevated expression of sFas protein in tumor cells causing apoptotic resistance via a different mechanism from the previously described three mechanisms (5).

The analysis of Fas and sFas protein expression is essential for the evaluation of potential pathological alterations of apoptotic pathways in pulmonary adenocarcinomas. In this study, we evaluated Fas and sFas protein expression in surgically resected human pulmonary adenocarcinomas and examined the correlation between the expression of these proteins and clinical prognosis. We also evaluated potential mechanisms for the loss of Fas protein expression and Fas-mediated apoptosis in pulmonary adenocarcinoma cell lines.

\section{Methods}

Patient characteristics. Written consent was obtained from 39 patients $(20$ males, mean age of $61.9 \mathrm{yr}$, and 19 females, mean age of $64.2 \mathrm{yr}$ ) who underwent pulmonary resection for lung cancer at the University of Michigan Medical Center from May 1991 to May 1994.

1. Abbreviation used in this paper: $\mathrm{sFas}$, soluble form of the Fas protein. 
These studies were approved by The University of Michigan Institutional Review Board. Three patients with stage I tumors had synchronous pulmonary cancers (both adenocarcinoma in each case). All 42 tumors were diagnosed as primary pulmonary adenocarcinomas according to criteria of the World Health Organization (10), and included four bronchioloalveolar cell carcinomas. The 42 adenocarcinomas include: 9 well differentiated, 10 moderately differentiated, and 23 poorly differentiated adenocarcinomas. 27 tumors were stage I, 2 were stage II, and 13 were stage IIIA. Surgical staging was determined using criteria defined by the American Joint Committee on Cancer (11). Clinical prognosis was also evaluated in 26 stage I and stage II patients who underwent operations from May 1991 to May 1993, and 13 stage IIIA patients who underwent operations from May 1991 to May 1994. The postoperative clinical course was evaluated for at least $4 \mathrm{yr}$ for stage I and stage II cancers and for $3 \mathrm{yr}$ for stage IIIA cancers. Clinical follow-up ranged from 10-64 mo (mean: 41.7) in stage I and stage II to 7-59 mo (mean: 32.8) in stage IIIA. 7 of 26 (26.9\%) patients of stage I and II and 5 of 13 (38.5\%) patients of stage IIIA patients died because of the recurrence of primary lung cancer during these follow-up periods. Patients were divided into two groups: alive (good prognostic cases) or dead (poor prognostic cases) based on their last follow-up date. Cases in which the cause of death was questionable were excluded.

Sample collection. Freshly harvested tissue samples from pulmonary resections were transported to the laboratory in DME (Life Technologies, Gaithersburg, MD) on ice. A portion of each tumor sample and nontumorous lung tissue was quickly frozen in liquid nitrogen for RNA and DNA isolation, and was stored at $-70^{\circ} \mathrm{C}$. Another portion of each sample was frozen in OCT compound (Miles, Elkhart, IN) using isopentane cooled to the temperature of liquid nitrogen for cryostat sectioning and immunohistochemical analysis.

Immunohistochemistry. 5- $\mu \mathrm{m}$ cryostat tissue sections were mounted onto poly-L-lysine-coated slides and then stored at $-20^{\circ} \mathrm{C}$. The slides were air dried for $10 \mathrm{~min}$ before fixation in $100 \%$ acetone at $-20^{\circ} \mathrm{C}$ for $10 \mathrm{~min}$. Endogenous peroxidase activity was inhibited using a $0.5 \%$ solution of $\mathrm{H}_{2} \mathrm{O}_{2}$ in $\mathrm{pH} 7.5 \mathrm{PBS}$ twice for $30 \mathrm{~min}$ each. Normal horse serum diluted 1:20 with PBS and containing 1.0\% BSA (PBSBSA) was incubated with the samples for $30 \mathrm{~min}$ to suppress nonspecific binding. Tissue sections were incubated with anti-Fas monoclonal antibody directed against $\mathrm{NH}_{2}$-terminal residues (clone DX-2; PharMingen, San Diego, CA) at 1:100 dilution in PBS-BSA for $1 \mathrm{~h}$ at room temperature. PBS-BSA was substituted for the primary antibody for the negative controls in each sample. Some specimens were also treated with an isotype-matched antibody to control for nonspecific staining (murine IgG1; PharMingen). Samples were then washed extensively in PBS and then incubated with a 1:1,000 dilution of biotinylated goat anti-mouse secondary antibody in PBS-BSA for $30 \mathrm{~min}$. The detection of bound antibody was accomplished using the avidinbiotin complex method with a mouse immunodetection kit (Vectastain ABC system; Vector Laboratories, Inc., Burlingame, CA). A $0.1 \%$ solution of $3,3^{\prime}$-diaminobenizidine in PBS was used as a chromogen. The slides were lightly counterstained with hematoxylin. In this study, Fas expression was also evaluated using anti-Fas polyclonal antibody which recognized $\mathrm{COOH}$-terminal residues (C-20; Santa Cruz Biotechnologies, Santa Cruz, CA). Immunohistochemical examination of all samples was performed independently by two reviewers.

Cell lines and flow cytometric analysis. The human lung adenocarcinoma cell lines A549 and A427 and the human lymphoma cell lines Jurkat and BJAB were used to examine Fas protein regulation. Jurkat and BJAB cells were used as positive controls of Fas-expressing cell lines. COS cells and MCF-7 cells were also used for transfection assays. These cell lines were obtained from American Type Culture Collection (Rockville, MD). The human lung adenocarcinoma cells, COS cells, and MCF-7 cells were cultured in DME containing $10 \%$ FBS and $1 \%$ penicillin/streptomycin, and the human lymphoma cell lines were cultured in RPMI 1640 (Sigma Chemical, St. Louis, MO) containing $10 \%$ FBS and $1 \%$ penicillin/streptomycin.
For FACScan ${ }^{\circledR}$ analysis, adherent adenocarcinoma lung cell lines were rinsed in PBS, incubated in $10 \mathrm{mM}$ EDTA incubated for $20 \mathrm{~min}$ at $4^{\circ} \mathrm{C}$, and harvested by gentle pipetting. After three washes in PBS, the A549, A427, and BJAB cells $\left(10^{6}\right.$ cells/200 $\left.\mu \mathrm{l}\right)$ were resuspended in flow cytometry buffer (PBS with $0.5 \%$ rabbit serum and $10^{-3} \mathrm{mM}$ $\mathrm{NaN}_{3}$ ), and labeled for $30 \mathrm{~min}$ at $4^{\circ} \mathrm{C}$ with anti-Fas primary antibody (clone DX-2; PharMingen) diluted with $200 \mu \mathrm{l}(1.25 \mu \mathrm{g} / \mathrm{ml})$ of flow cytometry buffer. After washing with flow cytometry buffer, the cells were labeled with FITC-conjugated anti-mouse secondary antibody (Biosource International, Camarillo, CA) diluted to 1:1,000 in flow cytometry buffer. Flow cytometric analysis was carried out using a FACScan ${ }^{\circledR}$ (Becton Dickinson, San Jose, CA) gated to exclude fractured cells and debris. 10,000 cells were examined for each determination.

Anti-Fas antibody-mediated apoptosis. Cell lines were plated in 96-well plates at $4 \times 10^{4}$ cells/well and allowed to adhere overnight. After replacing the media, cells were incubated with $12.5 \mu \mathrm{g} / \mathrm{ml}$ of DX-2 anti-Fas antibody and $20 \mu \mathrm{g} / \mathrm{ml}$ recombinant protein G (Sigma Chemical Co.) for $20 \mathrm{~h}$. Wells containing no treatment or recombinant protein $\mathrm{G}$ alone served as controls. Adherent and nonadherent cells were harvested, washed in PBS, and incubated at $4^{\circ} \mathrm{C}$ for $12 \mathrm{~h}$ in a propidium iodide-containing cell lysis buffer in which nuclei remained intact $(0.1 \%$ sodium citrate, $0.1 \%$ Triton X-100, and $10 \mu \mathrm{g} / \mathrm{ml}$ propidium iodide). A FACScan ${ }^{\circledR}$ flow cytometer was used to quantify the percentage of cells undergoing apoptosis as described previously $(12,13)$. Briefly, the fluorescence of propidium iodide in each nuclei was plotted histographically and nuclei which fluoresced below the peak representing G1 DNA content were counted as being apoptotic and reported as a percentage of the total number of nuclei counted (5,000 gated events).

Isolation of DNA, RNA, and protein. Genomic DNA was isolated from tumor and nontumorous tissues as described previously (14). RNA was extracted using the Trizol reagent (Life Technologies) as recommended by the supplier. Protein was extracted from tissues and cells by detergent lysis using NP-40 lysis buffer ( $0.2 \%$ NP- $40,100 \mathrm{mM}$ Tris-HCl, pH 8.0, $200 \mathrm{mM} \mathrm{NaCl}, 0.01 \%$ SDS).

Southern blot hybridization. Genomic DNA $(10 \mu \mathrm{g})$ of lung tumor, nontumorous lung tissues, and A549 and A427 cells were subjected to overnight digestion with the restriction enzyme EcoRI $(5 \mathrm{U} / \mu \mathrm{g})$ at $37^{\circ} \mathrm{C}$. The digested DNA was size-fractionated in $0.9 \%$ agarose gels containing ethidium bromide and transferred to nylon membranes. The blots were hybridized overnight with a random-primed (Life Technologies), $\left[\alpha^{-}{ }^{32} \mathrm{P}\right] \mathrm{dCTP}-$ labeled (Amersham, Arlington Heights, IL) human Fas cDNA fragment at $42^{\circ} \mathrm{C}$ in $50 \%$ formamide, $5 \times \mathrm{SSPE}$ (0.9 $\mathrm{M} \mathrm{NaCl}, 50 \mathrm{mM} \mathrm{NaH} \mathrm{PO}_{4}, \mathrm{pH} 7.7,5 \mathrm{mM}$ EDTA), 5\% dextran sulfate, $1 \times$ Denhardt's solution, $3 \%$ SDS, and $100 \mu \mathrm{g} / \mathrm{ml}$ of denatured salmon sperm DNA ( $\mathrm{pH}$ 7.0). The human Fas cDNA was generously provided by Dr. V.M. Dixit (Ann Arbor, MI). The blots were washed in $2 \times$ SSC $(20 \times$ SSC: $0.9 \mathrm{M} \mathrm{NaCl}, 0.3 \mathrm{M} \mathrm{Na}$ citrate $)$ and $0.1 \%$ SDS at $65^{\circ} \mathrm{C}$, and $0.1 \times \mathrm{SSC}$ at room temperature and then exposed to $\mathrm{x}$-ray film (Hyperfilm-MP; Amersham) with intensifying screens at $-70^{\circ} \mathrm{C}$.

Northern blot hybridization. Samples of total RNA (10 $\mu \mathrm{g})$ isolated from lung tumor, nontumorous lung tissues, and the A549 cell line were size-fractionated in $1.2 \%$ agarose, $2.2 \mathrm{M}$ formaldehyde gels and transferred to nylon membranes. The blots were hybridized at $48^{\circ} \mathrm{C}$ in $50 \%$ formamide, $5 \times$ SSPE, $5 \%$ dextran sulfate, $1 \times$ Denhardt's solution, $3 \%$ SDS, $100 \mu \mathrm{g} / \mathrm{ml}$ of denatured salmon sperm DNA, and $60 \mu \mathrm{g} / \mathrm{ml}$ of yeast tRNA (pH 7.0) containing the $\left[\alpha^{-32} \mathrm{P}\right]-$ dCTP-labeled human Fas cDNA probe described above. The blots were washed in $2 \times \mathrm{SSC}$ and $0.1 \%$ SDS at $60^{\circ} \mathrm{C}$, and $0.1 \times \mathrm{SSC}$ at room temperature and then exposed to Hyperfilm-MP with intensifying screens at $-70^{\circ} \mathrm{C}$. The intensity of Fas mRNA signals were analyzed using a scanning laser densitometer (Molecular Dynamics, Sunnyvale, CA). The loading and transfer of total RNA were normalized using a ${ }^{32} \mathrm{P}$-labeled oligonucleotide probe for the 28S rRNA as previously described (15).

$R T$-PCR, genomic PCR, and sequence analysis. First-strand cDNAs 
were synthesized from $10 \mu \mathrm{g}$ of total RNA in $40-\mu l$ reactions using oligo (dT) primers (Pharmacia, Piscataway, NJ) in the presence of avian myeloblastosis virus reverse transcriptase (Promega, Madison, WI) under conditions described by the supplier. First-strand cDNA $(8 \mu \mathrm{l})$ was amplified using Taq DNA polymerase (Promega) in a 100- $\mu \mathrm{l}$ reaction volume under standard conditions recommended by the manufacturer. The mixture was denatured for $5 \mathrm{~min}$ at $94^{\circ} \mathrm{C}$ followed by 35 thermal cycles with each cycle representing $1 \mathrm{~min}$ at $94^{\circ} \mathrm{C}, 1 \mathrm{~min}$ at $52^{\circ} \mathrm{C}$, and $2 \mathrm{~min}$ at $72^{\circ} \mathrm{C}$. Oligonucleotide primers for PCR amplification were derived from published sequences (16). For the transmembrane Fas cDNA analysis, the $5^{\prime}$ primer (5'-CATGGCTTAGAAGTGGAAAT-3') and $3^{\prime}$ primer (5'-ATTTATTGCCACTGTTTCAGG- $3^{\prime}$ ) were used. For the full-length sequence Fas cDNA analysis, the $5^{\prime}$ primer for Fas (5'-GTCGACCACTTCGGAGGATTGCTCAAC-3') was linkered with an SalI restriction enzyme site and the $3^{\prime}$ primer for Fas (5'-CTCTAGACTAGACCAAGCTTTGGATTTC-3') was linkered with an XbaI restriction enzyme site. The PCR products of the full-length Fas cDNA were ligated into a pED expression vector (17).

Genomic DNA $(1 \mu \mathrm{g})$ was amplified using Taq DNA polymerase (Promega) in a $100-\mu$ l reaction volume under standard conditions recommended by the manufacturer. The mixture was denatured for 5 min at $94^{\circ} \mathrm{C}$ followed by 35 thermal cycles with each cycle representing $1 \mathrm{~min}$ at $94^{\circ} \mathrm{C}, 1 \mathrm{~min}$ at $55^{\circ} \mathrm{C}$, and $2 \mathrm{~min}$ at $72^{\circ} \mathrm{C}$. Oligonucleotide primers for PCR amplification of exon 1 of Fas were derived from the published sequence (18), and were $5^{\prime}$ primer (5'-TTTGTGCAACGAACCCTGACTCCT-3') and $3^{\prime}$ primer (5'-GACTAAGACGGGGTAAGCCTCCAC-3'). The PCR products were purified and sequenced by automated fluorescent DNA sequencing (Applied Biosystems) at the Sequencing Core Facility of The University of Michigan.

Transfection. The expression vector pcDNA3 (Invitrogen, Carlsbad, CA) containing a 1.0-kb human Fas cDNA was used for stable transfection assays $(19)$. A549 cells $\left(3 \times 10^{6}\right)$ were transfected with $2 \mu \mathrm{g}$ plasmid DNA and Lipofectin (Life Technologies) using the protocol recommended by the supplier. Selection with G418 $(600 \mu \mathrm{g} / \mathrm{ml}$; Life Technologies) was initiated $48 \mathrm{~h}$ after transfection. Single clones were obtained 3-4 wk after transfection, expanded, and further characterized for Fas expression by Northern blot hybridization, flow cytometry, and Fas-mediated apoptosis using 96-well plate cytotoxicity assays as described previously (20).

The pcDNA3 expression vector containing the 1.0-kb human FasFLAG (FLAG epitope $\mathrm{NH}_{2}$-terminal) fusion protein cDNA (kindly provided by Dr. V.M. Dixit) was used for transient transfection assays (21). A549 cells $\left(3 \times 10^{6}\right)$ were transfected with $2 \mu \mathrm{g}$ plasmid DNA using Lipofectin (Life Technologies). The location of the FasFLAG protein was analyzed using an anti-FLAG monoclonal antibody and confocal microscopy.

The pED expression vector containing the full-length Fas cDNA amplified from the A549 and Jurkat cell lines was transiently transfected into COS cells using $10 \mu \mathrm{g}$ of plasmid DNA and the DEAE dextran (Pharmacia) method as previously described (22). $48 \mathrm{~h}$ after transfection, Fas expression was evaluated by both flow cytometry and metabolic labeling using $\left.{ }^{[5} \mathrm{S}\right]$ methionine and cytosine (23).

Confocal microscopy. Cells (A549 and MCF-7) grown on slides were transiently transfected with the FLAG-tagged Fas expression vector $(10 \%$ efficiency, nontransfected cells served as a control for staining specificity) and fixed in $100 \%$ acetone at $-20^{\circ} \mathrm{C}$ for $10 \mathrm{~min}$ after $48 \mathrm{~h}$. Nonspecific binding was blocked using a 1:20 dilution of goat serum in PBS. Fas protein was detected using a monoclonal antiFLAG antibody (clone M2; Eastman Kodak, Rochester, NY) at a 1:500 dilution. Immunoreactivity was detected using a secondary goat anti-mouse IgG FITC monoclonal antibody (Biosource International) at a 1:250 dilution. Coverslips were mounted with Fluoromount-G (Southern Biotechnology Associates, Birmingham, AL). Images were obtained using a confocal microscope (Bio-Rad, Hercules, CA)

Western blot hybridization. Samples of tissue and cellular protein
$(10 \mu \mathrm{g})$ were boiled for $10 \mathrm{~min}$ in an equal volume of sample buffer (20\% glycerol, $4 \%$ SDS, $0.2 \%$ bromophenol blue, $125 \mathrm{mM}$ Tris- $\mathrm{HCl}$, $150 \mathrm{mM} \mathrm{NaCl}, 5 \mathrm{mM}$ EDTA, $640 \mathrm{mM} \beta$-mercaptoethanol), and sizefractionated using $12 \%$ SDS-PAGE. Proteins were transferred to nylon membranes, and the Fas protein was detected using an anti-Fas rabbit polyclonal antibody (C-20; Santa Cruz Biotechnologies) at a 1:200 dilution. A goat anti-mouse monoclonal antibody conjugated with horseradish peroxidase (Chemicon, Temecula, CA) was used at a 1:2,000 dilution to visualize immunoreactive proteins using enhanced chemiluminescence (Pierce, Rockford, IL) and HyperfilmMP as recommended by the manufacturer.

Statistical analysis. Statistical analyses were performed with a Stat View 4.02 program package (Abacus Concepts, Berkeley, CA). The significances of the associations were determined by using the $\chi^{2}$ test, Fisher's exact probability test, or a two-tailed unpaired $t$ test with significance determined for $P<0.05$. Univariate and multivariate analyses of lung cancer patient survival data were undertaken by using survival curves and applying the Kaplan-Meyer method with log rank analysis.

\section{Results}

Fas protein expression in lung adenocarcinomas. Using an $\mathrm{NH}_{2}-$ terminal anti-Fas antibody, Fas protein expression was observed in $47.6 \%(20 / 42)$ of all lung adenocarcinomas and in $48.7 \%$ (19/39) of all patients. Eight tumor samples comprising four Fas protein-positive and four Fas protein-negative tumors, as determined using an $\mathrm{NH}_{2}$-terminal specific anti-Fas antibody (clone DX-2), were also examined with a COOH-terminal specific polyclonal anti-Fas antibody (C-20). Similar staining results were obtained with both antibodies. In three synchronous pulmonary adenocarcinoma patients, two cases demonstrated Fas expression in both tumors and the other case showed positive and negative Fas expression in each tumor. This later case was excluded from staging and prognostic analysis. Fas protein expression was observed in $48.2 \%(13 / 27)$ of stage I tumors, $100 \%(2 / 2)$ of stage II tumors, and in $38.5 \%$ $(5 / 13)$ of stage IIIA tumors. Fas protein expression was also observed in $22.2 \%$ (2/9) of well differentiated adenocarcinomas, in $60.0 \%(6 / 10)$ of moderately differentiated adenocarcinomas, and in $52.2 \%(12 / 23)$ of poorly differentiated adenocarcinomas. The incidence of Fas protein expression in poorly and moderately differentiated adenocarcinoma was higher than that of well differentiated adenocarcinoma, but this difference was not statistically significant. Fas protein-positive tumors and the lung adenocarcinoma cell line A549 demonstrated a diffuse cytoplasmic staining pattern without detectable membranous staining (Fig. 1). Fas protein expression was observed in 50.0\% (13/26) of alive patients and in $41.7 \%(5 / 12)$ of dead patients. The Kaplan-Meyer survival analysis using the log rank test showed no statistical difference in survival between patients with Fas protein-positive tumors and patients with Fas protein-negative tumors when grouped together regardless of stage, or when matched for stage $(P>0.1)$ (comparisons for stage II alone were not feasible).

Southern blot analysis. Southern blot analysis of the Fas gene in eight cases of pulmonary adenocarcinoma (three Fas protein-negative and five Fas protein-positive) and two pulmonary adenocarcinoma cell lines of A549 and A427 was performed. The primary tumor and nontumorous lung samples for each patient were examined. Three Fas bands were present in all samples, and no differences between Fas protein-negative and Fas protein-positive tumors, or in A549 and A427 

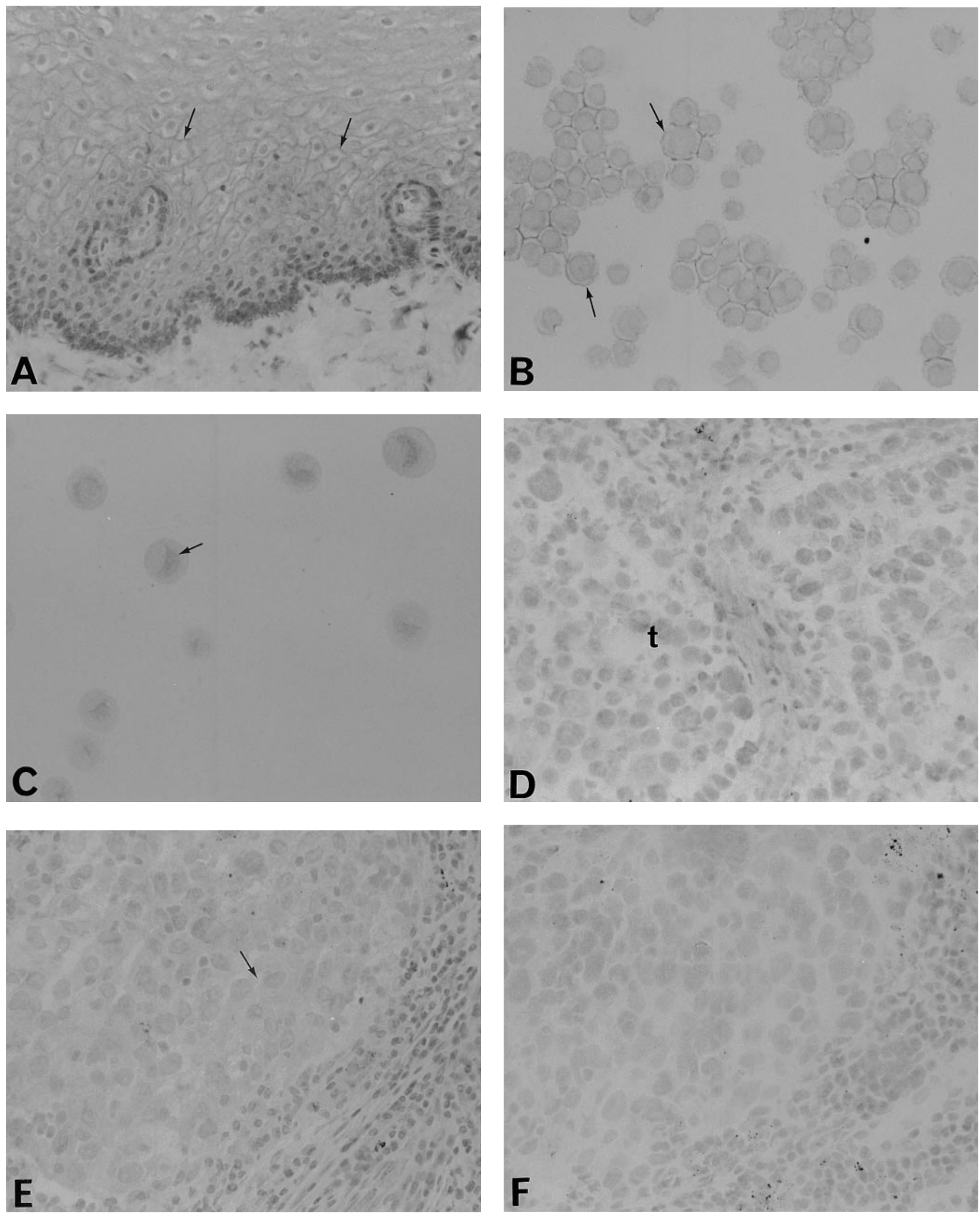

Figure 1. Immunohistochemical demonstration of membranous Fas protein expression (arrows) in $(A)$ the basal region of normal squamous esophageal epithelium and $(B)$ the BJAB cell line. $(C)$ In contrast, Fas protein expression appears localized within the cytoplasm in the pulmonary adenocarcinoma cell line A549. (D) A Fas-negative pulmonary adenocarcinoma with tumor cells $(t)$ showing no Fas protein immunoreactivity. (E) A Fas-positive pulmonary adenocarcinoma demonstrating a diffuse cytoplasmic Fas expression pattern in the tumor cells (arrow) without cell surface staining and $(F)$ a near-serial section of the same tumor shown in $E$ treated with an isotype-matched antibody indicating the anti-Fas antibody staining is specific. All cryostat sections were lightly counterstained with hematoxylin. Original magnifications, $\times 200$. 

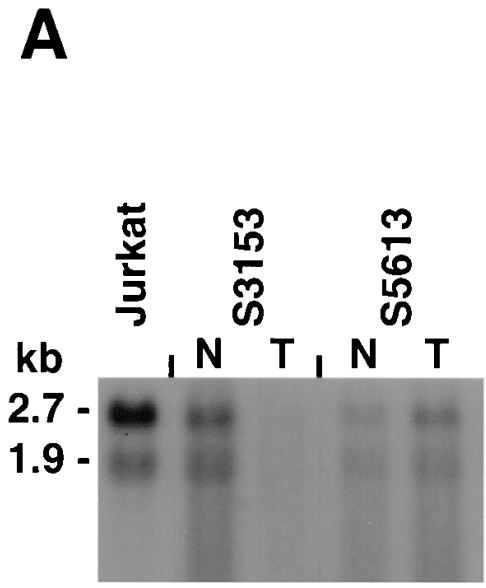

kb

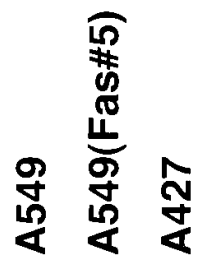

28S
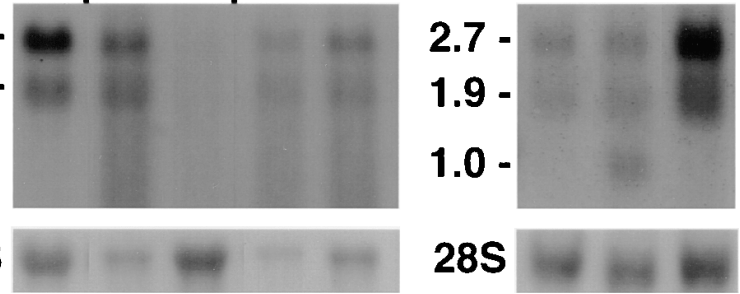

Figure 2. (A) Northern blot analysis demonstrating low level expression of Fas mRNA in a Fas protein-negative lung tumor $(T)$ (S3153), but Fas mRNA expression is present in a Fas protein-positive lung tumor $(T)$ (S5613) relative to the nontumorous lung $(N)$ from each patient. The results from all patients are summarized in Table I. (B) Northern blot analysis demonstrating abundant Fas mRNA expression in A549 and A427 cells. A549 cells stably transfected with a Fas cDNA express an additional $1.0 \mathrm{~kb}$ Fas mRNA. The $28 \mathrm{~S}$ rRNA signals are shown in the lower panels.

cells were detected (data not shown). These results indicate that major alterations or deletions of the Fas gene are not responsible for the lack of Fas protein expression in these tumors.

Fas $m R N A$ expression and sequence analysis. Northern blot analysis was evaluated in eight cases of pulmonary adenocarcinoma and in the cell lines A549, A427, Jurkat, and BJAB. Four of the tumors were Fas protein-negative and four tumors were Fas protein-positive as determined by immunohistochemistry. Both Jurkat and BJAB human lymphoma cell lines demonstrated two Fas mRNA bands (2.7 and $1.9 \mathrm{~kb})$ as previously described (16), and both A549 and A427 cell lines also demonstrated marked Fas mRNA expression (Fig. 2, $A$ and $B)$. Tumor and nontumorous lung samples in all eight cases also demonstrated Fas mRNA expression. The intensity of both Fas mRNAs were evaluated by densitometry after nor-

Table I. Fas mRNA Expression in Tumor and

Nontumorous Tissues

\begin{tabular}{lccc}
\hline Patient & Fas protein & Tumor & Nontumorous lung \\
\hline S3153 & - & 0.0916 & 3.1026 \\
H3833 & - & 0.3291 & 2.1182 \\
P2210 & - & 0.3443 & 1.4566 \\
R6777 & - & 0.3659 & 0.5243 \\
H5754 & + & 0.3508 & 0.3599 \\
B2103 & + & 1.1622 & 5.1360 \\
S5613 & + & 1.4685 & 2.1824 \\
F9510 & + & 2.6055 & 1.9771
\end{tabular}

Presented values represent the intensity of Fas mRNA/the intensity of 28S rRNA signals. malization with the $28 \mathrm{~S}$ rRNA signal (Table I). The intensity of the Fas/28S RNA ratio in Fas protein-positive tumors was higher than in Fas protein-negative tumors, but did not reach statistical significance $(P=0.056)$. There was also no statistical difference in the ratios of Fas/28S in nontumorous lung samples between Fas protein-positive and -negative cases $(P>0.1)$.

The mRNA of soluble Fas represents a transcript which is missing the transmembrane domain of exon 6. This soluble Fas mRNA is 63 bp shorter than native Fas mRNA. Because this small size difference cannot be distinguished by Northern blot analysis, we evaluated soluble Fas mRNA expression using RT-PCR. Of seven Fas protein-positive tumors determined by immunohistochemistry, all seven samples demonstrated the correct-sized PCR products for the native-form Fas mRNA (338 bp). These RT-PCR products were confirmed to be Fas by production of 175- and 163-bp bands after restriction enzyme digestion with BamH1. Two of the Fas protein-negative samples also demonstrated the correct-sized RT-PCR products (338 bp). Four of nine tumor samples showed a 63-bp smaller-sized RT-PCR product (275 bp) which represents the soluble form of Fas mRNA (Fig. 3), and this was confirmed by the absence of a BamH1 restriction site. These four sFas mRNAs were detected in one Fas-negative and three Fas-positive tumors. Within reactions resulting in two different product sizes, the smaller-sized sFas mRNA signals were much weaker than those of the native Fas mRNA signal. For all nine tumor samples, RT-PCR was also used to evaluate nontumorous lung from these patients for sFas expression and in all, there were positive RT-PCR products for the native Fas mRNA (338 bp), but not the soluble Fas mRNA (Fig. 3). The A549 and A427 also demonstrated strong PCR products (338 bp) of native Fas mRNA with very faint RT-PCR products (275 bp) of the soluble Fas mRNA. We evaluated DNA sequence analysis in the $5^{\prime}$ region of the Fas cDNA and in the noncoding region of the Fas gene (exon 1) of A549 cells; however, mutational changes as compared with the published sequences were not detected in either analysis (data not shown).

Western blot analysis. Western blot analysis was used to evaluate six primary lung tumor samples and the A549 and A427 cell lines. In the six tumors, of which four were Fas protein-positive and two were negative, all six tumor samples and both cell lines demonstrated a $48-\mathrm{kD}$ Fas protein band. A very faint smaller band ( $45 \mathrm{kD})$, which may either represent soluble Fas or nonspecific band, was present in all of these samples (Fig. 4). A smaller-sized band (42 kD), which represents the nonglycosylated primary translated Fas protein, was observed

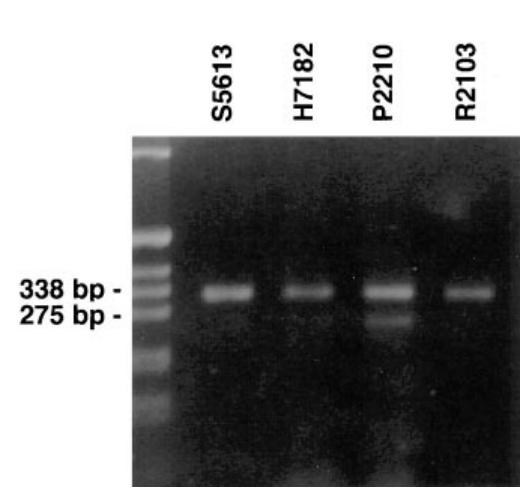

Figure 3. RT-PCR of Fas mRNA produces a 368-bp product in all seven tumors and nontumorous lung tissues. A smaller soluble Fas mRNA (275 bp) is observed in four of the tumor tissues. One Fas protein-positive tumor (P2210) demonstrates marked soluble Fas mRNA as shown by a 275-bp PCR product. 


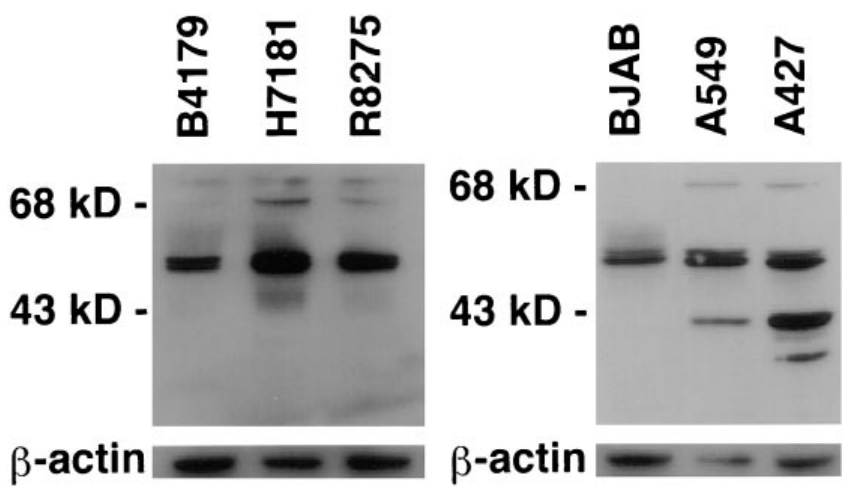

Figure 4. Western blot analysis demonstrating Fas protein (48 kD) expression in both Fas protein-positive (H7181 and R8275) and negative (B4181) lung tumors. A faint soluble Fas band at $45 \mathrm{kD}$, such as that present in the tumor from patient H7181, was only observed in a few samples. Abundant Fas protein $(48 \mathrm{kD})$ was detected in both A549 and A427 lung adenocarcinoma cells. The presence of a smaller band $(42 \mathrm{kD})$ in these cells is consistent with nonglycosylated Fas protein. Protein size markers are shown on the left and $\beta$-actin is shown in the lower panels.

in the A549 and A427 cell lines. Another smaller-sized band (35 kD) was present in the A427 cell lines; however, this band was not reproducibly observed.

Surface expression of Fas protein. In the cell lines, surface expression of the Fas protein was evaluated using FACScan ${ }^{\circledR}$ analysis. The BJAB cell line was used as positive control of surface Fas protein expression. We applied the mean fluorescence intensity of the population of cells as a measure of relative surface Fas protein expression (20). The mean fluorescence intensity of BJAB cells was 359.1, but was only 45.94 in A549 and 88.17 in A427 cells. Therefore, although Western blot analysis demonstrated abundant Fas protein expression in the A549 cell line, there was little surface Fas protein expression as measured by flow cytometry (Fig. $5 \mathrm{~A}$ ). Treatment of the A549 cells with an anti-Fas antibody also failed to induce apoptosis, indicating a lack of responsiveness to this antibody (Fig. 5 B). The A549 cell line expresses both Fas mRNA and Fas protein in Northern and Western blot analyses respectively; however, these cells appear to have negligible surface Fas protein expression and are resistant to Fas-mediated apoptosis.

Transfection of Fas. Fas cDNAs from both Jurkat (control) and the A549 cell lines were produced by RT-PCR and incorporated into the $\mathrm{pED}$ expression vector. The cDNAs were transiently transfected into COS cells, and surface Fas protein expression was evaluated by FACScan ${ }^{\circledR}$ analysis. The mean fluorescence intensity of COS cells treated with DEAE dextran alone was 45.8, 285.37 in COS cells transfected with the cDNA derived from Jurkat cells, and 286.86 in COS cells transfected with the cDNA derived from A549 (Fig. 5 C). This indicated that the transgenic expression in COS cells of a Fas cDNA derived from A549 resulted in the surface expression of Fas protein, and therefore suggested that the A549 Fas coding sequence was the wild type. Fas protein expression was also confirmed by metabolic labeling using $\left[{ }^{35} S\right]$ methionine and cytosine in COS cells transfected with A549 Fas cDNA (data not shown). We also evaluated 12 individual clones of A549 cells which were selected for neomycin resistance after transfection with the native Fas cDNA and then analyzed for surface Fas expression using FACScan ${ }^{\circledR}$ analysis. The mean fluorescence intensity of 2 of these 12 clones (99.2 and 101.7) was found to be approximately twofold greater than the mean fluorescence intensity of the nontransfected A549 cells which served as the control (45.94). Northern blot analysis was used to examine the level of Fas mRNA in these A549 stable transfectants. Both clones expressed the native Fas $(1.7$ and $2.9 \mathrm{~kb})$ and the transgenically expressed Fas (1.0 kb) mRNAs (Fig. 2 B); however, similar to the parental A549 cell line, the rate of apoptosis in these two clones after anti-Fas antibody treatment did not differ from the nontreatment controls. Therefore, only a minimal increase in surface Fas expression was identified in these neomycin-resistant A549 clones which were confirmed to be transcribing the transfected sequence by Northern blot analysis, yet remained resistant to Fas-mediated apoptosis.

Transient transfection of a FLAG-tagged Fas cDNA into A549 cells was used to confirm translation of the expression vector mRNA and to evaluate the localization of this transgenically expressed Fas protein. Using confocal microscopy and an anti-FLAG antibody, the Fas-FLAG protein was visualized within the cytoplasm of A549 cells; no Fas-FLAG protein was observed on the cell surface membranes (Fig. 6). These findings suggest that expression of the native Fas protein or transgenically expressed Fas protein by A549 cells does not result in sufficient levels of surface Fas protein expression to render these cells sensitive to Fas-mediated apoptosis.

\section{Discussion}

Fas plays a key role in apoptotic pathways $(1,4)$ and is expressed in various normal cells; however, the lack of Fas protein in malignant cells has been reported $(5,24,25)$. In Fasnegative malignant cells, the lack of Fas protein is considered as the primary mechanism for apoptotic resistance and insensitivity to antitumor treatments $(5,24)$. In the respiratory system, ciliated cells and goblet cells of the trachea, as well as alveolar cells, normally express the Fas protein (26). In a previous study (26), four of five bronchioloalveolar cell carcinomas and none of two pulmonary adenocarcinomas expressed the Fas protein. In our study, 2 of 4 bronchioloalveolar cell carcinomas $(50.0 \%)$ and 20 of 42 total pulmonary adenocarcinomas $(47.6 \%)$ expressed Fas. This incidence of Fas protein-positive tumors in pulmonary adenocarcinomas is similar to a previous report (26). The Fas protein traverses the cell surface membrane and is a receptor protein for the Fas ligand. In our study, Fas protein-positive cases demonstrated a diffuse cytoplasmic pattern without cell surface staining. This suggests that most pulmonary adenocarcinomas do not express Fas on their cell surface.

We found no significant correlation between Fas protein expression and patient prognosis. This may be due to an insufficient number of cases available for examination to detect any correlations between Fas protein expression and prognosis in pulmonary adenocarcinoma, or that the detection of the Fas protein using immunocytochemistry may not correlate with the biological function of Fas receptor.

Other genetic alterations that affect apoptotic resistance may occur in lung adenocarcinoma (27), one of which is bcl-2 protein overexpression. Using immunohistochemical analysis, the bcl-2 protein was visualized in $16.7 \%$ (7/42) of these tu- 

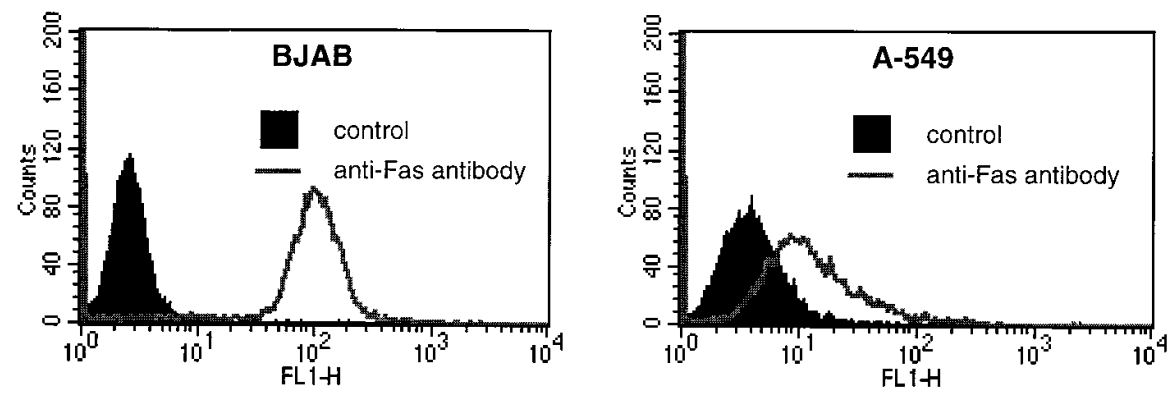

A

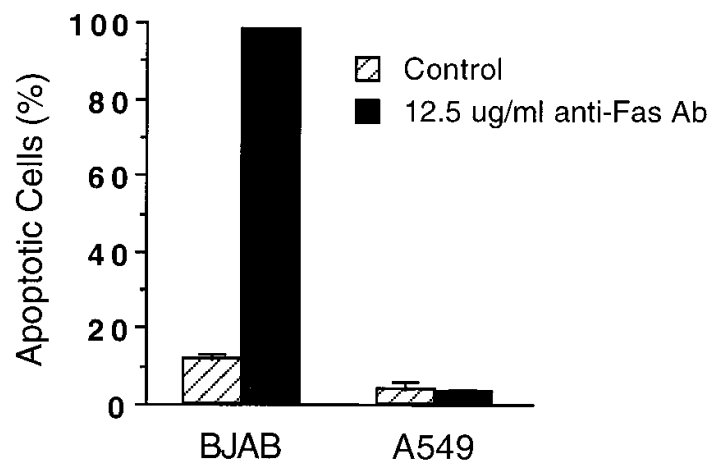

B
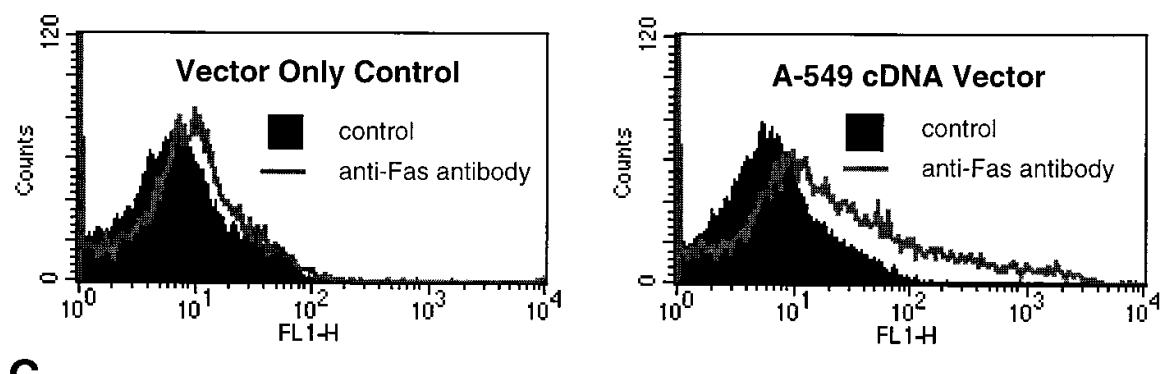

Figure 5. Surface Fas protein expression as measured by flow cytometry. The cell populations are those with the FITC-conjugated secondary antibody alone (gray fill) and with anti-Fas antibody (without fill). (A) A549 cells (48\%) express Fas protein, but levels of cell surface expression of Fas protein are significantly lower than the BJAB cells. (B) Percentage of cells undergoing apoptosis $20 \mathrm{~h}$ after treatment with anti-Fas antibody cross-linked with protein $\mathrm{G}$, or control treatment of protein $\mathrm{G}$ alone. A549 cells are resistant to Fas-mediated apoptosis. (C) COS cells transiently transfected with an expression vector $\mathrm{pED}$ containing the A549 Fas cDNA demonstrate similar levels of surface Fas protein expression as COS cells transfected with an expression vector containing the Jurkat Fas cDNA (data not shown).

mors, but similar to Fas expression, there was no significant statistical correlation between bcl-2 protein expression and prognosis (our unpublished data). We could not clarify any significant prognostic factors in pulmonary adenocarcinomas as it relates to these apoptosis-related proteins by immunohistochemistry.

Southern blot analysis indicated that both Fas protein-positive and -negative cases retain an apparently normal Fas gene. In hematological malignancies, rearrangements of the Fas gene have been reported (28); however, in our study major gene deletions or alterations were not observed in the eight primary tumors and A549 and A427 cell lines. Northern blot analysis did demonstrate higher levels of an appropriately sized Fas mRNA in three of four Fas protein-positive cases. One Fas protein-positive and all four Fas-negative tumors also showed low levels of Fas mRNA, suggesting decreased transcription of Fas in these tumors. Therefore, a reduction in Fas mRNA may be the mechanism of reduced Fas protein expression in at least a subset of tumors which are Fas protein-nega- tive by immunohistochemical analysis. In surgically resected samples, normal Fas protein-expressing cells such as lymphocytes and alveolar cells are often present, and these cells may affect the levels of Fas mRNA observed by Northern blot analysis. However, the A549 and A427 lung adenocarcinoma cell lines demonstrated abundant Fas mRNA expression and both cell lines showed very little surface Fas protein expression as determined by flow cytometry. In a previous report of Fas protein expression in glioma cell lines (24), a significant correlation between surface Fas protein and mRNA expression was observed. Fas cDNA transfer into these glioma cell lines also induced surface Fas protein expression and susceptibility to anti-Fas treatment (24). In pulmonary adenocarcinoma, however, we could not identify a significant correlation between Fas mRNA expression and the cell surface expression of Fas.

The native Fas protein is a membrane-anchored receptor for the Fas ligand $(4,16)$, and the soluble forms of the Fas protein contain deletions within the transmembranous domain (68 ). These soluble forms of the Fas protein are easily released 

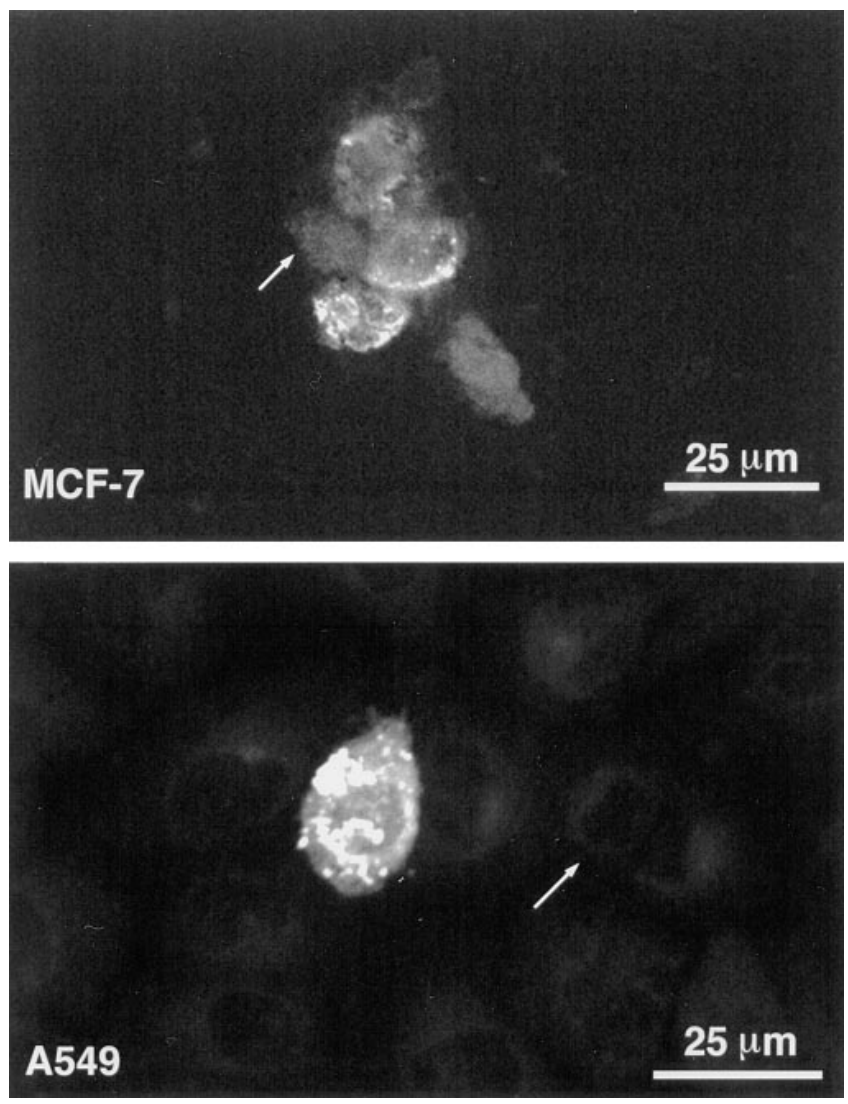

Figure 6. Confocal microscopy of A549 and MCF-7 cells transiently transfected with a FLAG-tagged Fas construct and examined using immunohistochemistry with an antibody to the FLAG epitope. The subset of A549 cells in which immunoreactivity was identified (bottom) demonstrate perinuclear and cytoplasmic staining without cell surface staining, whereas MCF-7 cells (top) show marked cell surface staining. Nontransfected cells (arrows) within each field confirm the specificity of the anti-FLAG antibody.

into the extracellular space and can neutralize the effect of the Fas ligand leading to tumor cell survival (6-8). Malignant cells which produce sFas protein have been reported (7), and elevated levels of sFas protein have been detected in the sera of patients with nonhematological malignancies (9). We evaluated sFas mRNA and protein by RT-PCR and Western blot analyses in the pulmonary tumors. In our study, native Fas mRNA was predominant in both tumors and nontumorous lung tissues; however, sFas mRNA was identified in 2 of 10 samples. RT-PCR evaluation is not quantitative; however, it is a sensitive method for mRNA detection. We conclude that significant sFas mRNA expression is not present in either the tumor or nontumorous lung tissues. Western blot analysis also supports the conclusion that sFas protein is not abundantly expressed in pulmonary adenocarcinomas.

The mechanism for the lack of surface Fas protein in the A549 cell line was further evaluated because A549 cells demonstrated little surface Fas protein, but Fas mRNA expression was observed and thus was similar to the pulmonary adenocarcinomas examined in this study. Western blot analysis surprisingly showed relatively abundant Fas protein expression in these cells. We used an $\mathrm{NH}_{2}$-terminal specific anti-Fas antibody for flow cytometry and a $\mathrm{COOH}$-terminal specific anti-
Fas antibody for our Western blot analysis; however, we also confirmed the expression of Fas protein by Western blot using the $\mathrm{NH}_{2}$-terminal anti-Fas antibody (data not shown). Southern, Northern, and Western blot analyses demonstrated that the Fas gene, its transcription, and translation were preserved in the A549 cell line. Transfection of the Fas cDNA from the A549 cell into COS cells, which induced surface Fas protein expression in COS cells, indicated that the Fas mRNA from A549 cells was normal. DNA sequence analysis of the untranslated 5' sequence of the Fas cDNA from A549 cells also indicated no mutational change was present in this region (data not shown). It has been reported that alterations in the $5^{\prime}$ untranslated region of the Fas gene and Fas mRNA might result in the lack of surface Fas protein expression (29). Neither mutational changes nor transcriptional abnormalities explain the lack of surface Fas protein expression in A549 cells. Transfection of the native Fas cDNA into the A549 cells failed to induce significant surface Fas protein expression or responsiveness to Fas-mediated apoptosis, and transient transfection of a Fas-FLAG fusion cDNA demonstrated that the Fas-FLAG protein was located in the cell cytoplasm. Importantly, the bcl-2 protein is not overexpressed by either A549 or A427 cell lines (our unpublished data). These findings suggest that the Fas protein is retained in the cytoplasm, potentially due to alterations in the posttranslational machinery of these cells.

The Fas protein translated from mRNA is transported to the membrane after posttranslational modifications which include: (a) signal peptide cleavage; $(b)$ chaperone-assisted folding; (c) addition of N-linked oligosaccharides; $(d)$ disulfide bond formation; $(e)$ hydroxylation of proline or lysine residues; $(f)$ oligomerization; and $(g)$ addition of glycosylphosphatidyl inositol anchor. Alteration or modification of one or more of these processes in the A549 cells is likely, and future studies will clarify the precise abnormality within these cells. It is possible that similar defects may be present in the primary lung adenocarcinomas as well.

Four mechanisms for a developing apoptotic resistance have been emphasized in malignant cells: $(a)$ lack of cell-surface Fas protein expression; $(b)$ synthesis of a protective protein such as the bcl-2 protein; (c) alterations in Fas intracellular signaling pathways; and $(d)$ production of a soluble Fas protein. Recently, Fas ligand expression in lung carcinomas has been proposed to play a role in allowing escape from immune surveillance (30). The lack of cell-surface Fas protein is an essential factor in providing resistance to Fas-Fas ligandinducible apoptosis. Previous studies have focused on transcriptional and translational mechanisms of Fas and Fas ligand-mediated apoptosis in malignant cells. Alterations in posttranslational modification, suggested by the present studies, may be an additional factor that allows tumor cell survival by evading the apoptotic pathway. Similar abnormalities in posttranslational modification of Fas have also been observed in human esophageal adenocarcinomas (31). The analysis of potential posttranslational abnormalities of the Fas protein in malignant cells will be essential for future gene therapy using Fas cDNA transfer.

\section{Acknowledgments}

This study was funded in part by American Cancer Society grant CN171, National Institutes of Health training grant CA09672-06 (to S.J. Hughes), and a grant from the Frederick A. Coller Society. 


\section{References}

1. Garfinkel, L. 1991. Cancer statistics and trends. In American Cancer Society Textbook of Clinical Oncology. A.I. Holleb, D.J. Fink, and G.P. Murphy, editors. American Cancer Society, Atlanta, GA. 1-6.

2. Wyllie, A.H., J.F.R. Kerr, and A.R. Currie. 1980. Cell death: significance of apoptosis. Int. Rev. Cytol. 68:251-306.

3. Thompson, C.B. 1995. Apoptosis in pathogenesis and genes and treatment of disease. Science. 267:1456-1461.

4. Nagata, S., and P. Goldstein. 1995. The Fas death factor. Science. 267: 1449-1456.

5. Owen-Schaub, L.B., R. Radinsky, E. Krunzel, K. Berry, and S. Yonehara. 1994. Anti-Fas on nonhematopoietic tumors: levels of Fas/APO-1 and bcl-2 are not predictive of biological responsiveness. Cancer Res. 54:1580-1586.

6. Cheng, J., T. Zhou, C. Liu, J.P. Shapiro, M.J. Brauer, M.C. Keifer, P.J. Barr, and J.D. Mountz. 1994. Protection from Fas-mediated apoptosis by a soluble form of the Fas molecule. Science. 263:1759-1762.

7. Owen-Schaub, L.B., L.S. Angelo, R. Radinsky, C.F. Ware, T.G. Genser, and D.P. Bartos. 1995. Soluble Fas/APO-1 in tumor cells: a potential regulator of apoptosis? Cancer Lett. 94:1-8.

8. Cascino, I., G. Fiucci, G. Papoff, and G. Ruberti. 1995. Three functional soluble forms of the human apoptosis-inducing Fas molecule are produced by alternative splicing. J. Immunol. 154:1157-1164.

9. Midis, G.P., Y. Shen, and L.B. Owen-Schaub. 1996. Elevated soluble Fas (sFas) levels in nonhematopoietic human malignancy. Cancer Res. 56:38703874 .

10. World Health Organization. 1988. The World Health Organization histological typing of lung tumors (second edition). Am. J. Clin. Pathol. 77:123136.

11. American Joint Committee of Cancer. 1992. Manual for Staging of Lung Cancer. O.H. Beahrs, D.E. Henson, R.V.P. Hutter, and B.J. Kennedy, editors. Lippincott, Philadelphia, PA. 115-119.

12. Nicoletti, I., G. Mgliorati, M.C. Pagliacci, F. Grignani, and C. Riccadi. 1991. A rapid and simple method for measuring thymocyte apoptosis by propidium iodide staining and flow cytometry. J. Immunol. Methods. 139:271-279.

13. Darzynkiewicz, Z., S. Bruno, G. Del Bino, W. Gorczyca, M.A. Hotz, P. Lassota, and F. Traganos. 1992. Features of apoptotic cells measured by flow cytometry. Cytometry. 13:795-808.

14. Buffone, G.J., and G.J. Darlington. 1985. Isolation of DNA from biological specimens without extraction with phenol. Clin. Chem. 31:164-165.

15. Hanson, L.A., E.O. Nuzum, B.C. Jones, A.M. Malikinson, and D.G. Beer. 1993. Expression of the glucocorticoid receptor and Ki-ras genes in urethan-induced mouse lung tumors and transformed cell lines. Exp. Lung Res. 17: 371-387.

16. Itoh, N., S. Yonehara, A. Ishii, M. Yonehara, S.-I. Mizushima, M. Sameshima, A. Hase, Y. Seto, and S. Nagata. 1991. The polypeptide encoded by the cDNA for human cell surface antigen Fas can mediate apoptosis. Cell. 66: 233-243.
17. Davies, M.V., and R.J. Kaufman. 1992. Internal translation initiation in the design of improved expression vectors. Curr. Opin. Biotechnol. 3:512-517.

18. Wada, N., M. Matsumura, Y. Ohba, N. Kobayashi, T. Takizawa, and Y. Nakanishi. 1995. Transcription stimulation of the Fas-encoding gene by nuclear factor for interleukin-6 expression upon influenza virus infection. J. Biol. Chem. 270:18007-18012.

19. Jaattella, M., M. Benedict, M. Tewari, J.A. Shayman, and V.M. Dixit 1995. Bcl-X and Bcl-2 inhibit TNF and Fas-induced apoptosis and activation of phospholipase A2 in breast carcinoma cells. Oncogene. 10:2297-2305.

20. Weller, M., K. Frei, P. Groscurth, P.H. Krammer, Y. Yonekawa, and A. Fontana. 1994. Anti-Fas/APO-1 antibody-mediated apoptosis of cultured human glioma cells. Induction and modulation of sensitivity by cytokines. J. Clin. Invest. 94:954-964.

21. Chinnaiyan, A.M., K. O'Rourke, M. Tewari, and V.M. Dixit. 1995. FADD, a novel death domain-containing protein, interacts with the death domain of Fas and initiates apoptosis. Cell. 81:505-512.

22. Kaufman, R.J. 1990. Vectors used for expression in mammalian cells. Methods Enzymol. 185:487-511.

23. Rehemtulla, A., and R.J. Kaufman. 1992. Preferred sequence requirements for cleavage of pro-von Willebrand factor by propeptide-processing enzymes. Blood. 79:2349-2355.

24. Weller, M., U. Malipiero, A. Rensing-Ehl, P.J. Barr, and A. Fontana. 1995. Fas/APO-1 gene transfer for human malignant glioma. Cancer Res. 55: 2936-2944.

25. Keane, M.M., S.A. Ettenberg, G.A. Lowrey, E.K. Russell, and S. Lipkowitz. 1996. Fas expression and function in normal and malignant breast cell lines. Cancer Res. 56:4791-4798.

26. Leithäuser, F., J. Dhein, G. Mechtersheimer, K. Koretz, S. Brüderlein, C. Henne, A. Schmidt, K-M. Debatin, P.H. Krammer, and P. Möller. 1993. Constitutive and induced expression of APO-1, a new member of the nerve growth factor/tumor necrosis factor receptor superfamily, in normal and neoplastic cells. Lab. Invest. 69:415-429.

27. Hockenberry, D., G. Nuñez, C. Milliman, R.D. Screiber, and S.J. Korsmeyer. 1990. Bcl-2 is an inner mitochondrial membrane protein that blocks programmed death. Nature. 348:334-336.

28. Xerri, L., C. Nadine, P. Park, and F. Birg. 1995. Search for rearrangements and/or allelic loss of the fas/APO-1 gene in 101 human lymphomas. Am J. Clin. Pathol. 104:424-430.

29. Cai, Z., R. Stancou, M. Korner, and S. Chouab. 1996. Impairment of Fas-antigen expression in adriamycin-resistant but not TNF-resistant MCF-7 tumor cells. Int. J. Cancer. 68:535-546.

30. Niehans, G.A., T. Brunner, S.P. Frizelle, J.C. Liston, C.T. Salerno, D.J. Knapp, D.R. Green, and R.A. Kratzke. 1997. Human lung carcinomas express Fas ligand. Cancer Res. 57:1007-1012.

31. Hughes, S.J., Y. Nambu, O.S. Soldes, D. Hamstra, A. Rehemtulla, M.D. Iannettoni, M.B. Orringer, and D.G. Beer. 1997. Fas/APO-1 (CD95) is not translocated to the cell membrane in esophageal adenocarcinoma. Cancer Res. 57:5571-5578 